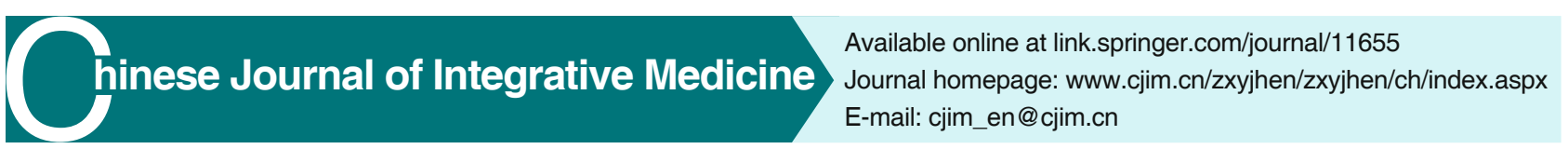

Perspective

\title{
A Formative History of Integrative Medicine in Hong Kong
}

\author{
YU Edwin Chau-leung and BIAN Zhao-xiang
}

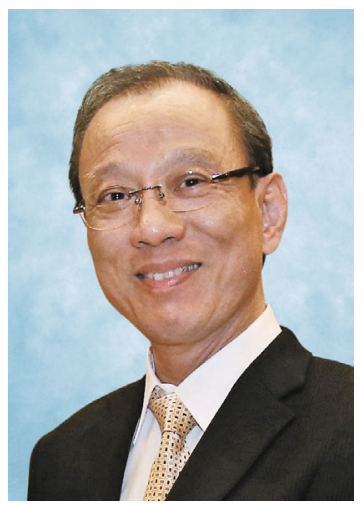

Prof. YU Edwin Chau-leung
With Chinese medicine

(CM) deeply rooted in Hong

Kong's Chinese culture, empirical practice of $\mathrm{CM}$ has been the norm since the early days of Hong Kong, China. Western medicine (WM) had its humble beginnings in Hong Kong in the form of established services for colonial government staff; WM was subsequently extended to the Chinese population through missionary efforts. The scourge of the plague pestilence in 1894 gave WM an advantage whence enforced laws and regulations and the swing to scientific philosophy set a trend that snowballed WM dominance. ${ }^{(1)}$

Since the late 19th century, Hong Kong residents have been seeking treatment from both CM and WM. CM was given an official status by the Basic Law in 1997. There came about the Chinese Medicine Ordinance in 1999 and the humble beginnings of integrative medicine (IM).

\section{Founding and Mission of Hong Kong Association for Integration of Chinese-Western Medicine}

In 1999, initiated by Dr. KO Wing-man and Dr. YU Edwin Chau-leung, a group of doctors with CM training gathered to study how CM could become useful in the Hong Kong medical scene. With the guidance of academic advisers, these doctors held meetings on various logistical, legal and academic aspects. Around this time, the University of Hong Kong formed a liaison with the Chinese Association of Integrative Medicine (CAIM) in Beijing. In 2001, spearheaded by Prof. CHOW Shew-ping, the former brainstorming group of Hong Kong doctors, scientists, and university professors in WM and $\mathrm{CM}$ joined hands and formed the Hong Kong Association for Integration of Chinese-Western Medicine (HKAIM). Since then, the HKAIM has led the development of IM in Hong Kong at four broad levels: (1) socio-economic-political level, (2) professional and bedside practice level, (3) education and training level, and (4) research and basic conceptual level.
The Association gained steady momentum under the leadership of its successive presidents. The founding president was Prof. Chow SP, after being Dean of the Faculty of Medicine and started serving as Pro-ViceChancellor of the University of Hong Kong (HKU). Prof. Chow was appointed a Justice of the Peace (JP) in 1995 and awarded the Silver Bauhinia Star in 2012. He is world-renowned for his contributions to orthopaedic surgery, particularly in upper limb surgery and microsurgery, with hundreds of publications and was appointed as honorary advisors and editors in international journals. Prof. Chow's clinical study in 1980s involved $\mathrm{CM}$ and confirmed acupuncture treatment superior to traditional medical methods for the sequelae of hand trauma. ${ }^{(2)}$ He also used animal experiment to confirm the promotion of fracture healing by the herb Drynaria fortunei. ${ }^{(3)}$ He pioneered the establishment of the School of Chinese Medicine (SCM) in HKU and afterwards led it as Chairman of the Management Committee. Prof. Chow is awarded Honorary University Fellow of HKU in 2018.

Dr. Ko WM was the second president (2004-2008). He served the position of Director of Professional Services and Public Affairs and then Director of Professional Services and Human Resources) during his time in the Hospital Authority (HA) of Hong Kong. During the severe acute respiratory syndrome (SARS) outbreak in 2003, he served $\mathrm{HA}$ as the acting Chief Executive. Dr. Ko was appointed as Secretary for Food and Health in the Hong Kong Government (2012-2017). He received the master's degree in health administration, fellowship from the Royal College of Surgeons of Edinburgh, and fellowship of the Faculty of Public Health Medicine at Royal College of Physicians. Dr. Ko was appointed as JP in 2001. For his remarkable contributions, he was awarded the Bronze

CThe Chinese Journal of Integrated Traditional and Western Medicine Press and Springer-Verlag GmbH Germany, part of Springer Nature 2019

Hong Kong Association for Integration of Chinese-Western Medicine, Hong Kong Baptist University, Hong Kong SAR, China Correspondence to: Prof. YU Edwin Chau-leung, E-mail: yuedwin@yahoo.com

DOI: https://doi.org/10.1007/s11655-019-3160-1 
Bauhinia Star in 2008 and Gold Bauhinia Star in 2017.

After him, the HKAIM presidency was succeeded by Dr. WONG TAAM Vivian Chi-woon (2008-2012). Dr. Wong is a college fellow of 3 disciplines: Obstetrics and Gynaecology, Internal Medicine and Public Health. She was Hospital Chief Executive of Queen Mary Hospital and worked once as Chief Executive in the HA. Dr. Wong was appointed JP in 1999 and received honorary fellowship from the Open University of Hong Kong in 2006 and awarded as Honorary University Fellow of HKU in 2007. Being Honorary Professor in SCM at the HKU, Dr. Wong offered and started the endowed professorship in IM in the SCM. She is most concerned with promoting ethical standards in the Clinical Trials Centre. She conducted a randomized controlled trial (RCT) on the effectiveness of acupuncture in induction of labor in the mid 1970s, and worked on the effect of qigong in cancer, diabetes and hypertension and the use of taichi to prevent fall in the elderly. With her experience as public health specialist in Policy and Research at the World Bank and Consultant to World Health organization (WHO), she is Advisor to the Good Practice in Traditional Chinese Medicine Research in the Post-genomic Era Consortium of the European Union and Co-chair for the Informatics Group of the Consortium for Globalization of Chinese Medicine (CGCM) and a member of the Board of Directors of Chinese Association of Integrative Medicine. She was instrumental when working as the CM Department of HA for the early development of the Chinese Medicine Centres for Training and Research (CMCTR).

Dr. Yu ECL became the fourth president (2012-2016) after being Founding Vice President over the years. He has been appointed as non-official member of the Chinese Medicine Development Committee of the Hong Kong SAR Government since 2013. Dr. Yu started the first hemodialysis, hemofiltration and plasmapheresis in children and neonates in the 1980s and was part of the medical team who performed the first successful kidney transplant to a child in Hong Kong. Currently spending about two-thirds of his time in clinic and mentoring medical students in InteMed HK, he is remarkable in Hong Kong as one of the few registered and practicing in both CM and WM at the same time in Hong Kong. He was awarded a Croucher Fellowship in 1987 and Fellowship of the Royal College of Physicians in 1989. He was awarded as Honorary Professor, SCM, Hong Kong Baptist University (HKBU) at its first listing. He serves with academic positions in the three universities. Dr. Yu has been elected as mentor in the Innovation and Technology Scholarship Award Scheme of the Innovation and Technology Commission (ITC), Hong Kong SAR. Dr. Yu was also the founder president for other academic societies including Paediatric Nephrology Society and Association for Integrative Aesthetic Medicine, Hong Kong. He has also been the Chairman in the Hong Kong Museum of Medical Sciences Society. With his passion in IM, Dr. Yu was elected as an Integrative Medicine Expert for the Chinese Association of Integrative Medicine in 2006. Dr. Yu expounded the CM concepts on Zang organs functions and dysfunctions in modern anatomico-functional terms, and won the Huaxia Medicine Prize for Excellent Paper in 2014. He is a prime mover to positioning $\mathrm{CM}$ having the pre-dominant role while collaborating with WM in the CM hospital. He was the Chief Coordinator for running the ITC-funded Integrative Joint Organizational Platform (2015-2017) and its continued program after 2019 to build a conducive environment for collaborative practice of CM and WM.

Currently these four presidents have become the Honorary Presidents of the HKAIM. The immediate past president is Prof. BIAN Zhao-xiang. He has been the Director of Clinical Division, SCM in HKBM. Prof. Bian is awarded Tsang Shiu Tim Endowed Chair of Chinese Medicine Clinical Studies. He is the Associate Dean and Chair Professor of Teaching and Research Division in $\mathrm{HKBU}$. He is instrumental in the development of the $\mathrm{CM}$ hospital in Hong Kong. His leadership drives up to the establishment of the HKBU Jockey Club Chinese Medicine Disease Prevention and Health Management Centre. He is also the Honorary Professor of the Shanghai University of Traditional Chinese Medicine and the China Academy of Chinese Medical Sciences. He has been awarded second prize of National Science and Technology Award of China in 1999. He is the reviewer of several grants, including the National Natural Science Foundation of China and Research Grant Council of Hong Kong, China. He also serves as a reviewer or editorial board member for many international journals. His main interest is on the relationship between psychological stress and digestive diseases, especially on colorectal cancer, irritable bowel syndrome (IBS) and inflammatory bowel disease (IBO) and related new drug discovery. Prof. Bian excels in clinical trial design, implementation and reporting with $\mathrm{CM}$, and these studies have been published in the Annals of Medicine.

After his presidency, starting 2018, the current president Dr. OR Ka-hang is also registered both in CM and WM in Hong Kong. Specialized in geriatrics and rehabilitation, he has been a consultant, and continues as a part time consultant to hospital authority for the research and informatics group in the HA. Dr. Or received fellowship of the Hong Kong Academy of Medicine and is a member 
of the Royal College of Physicians of Edinburgh. He is an Adjunct Associate Professor in the Chinese University of Hong Kong (CUHK). He has developed a whole line of rehabilitation treatment methods for the elderly, heart failure and stroke. He serves in various committees in the Food and Health Bureau (FHB), Chinese Medicine Council of Hong Kong, Innovation and Technology Fund and in the SCM in HKBU. Dr. Or has been the convener in Clinical Working Group for Stroke IM Management. He excels himself in research with $\mathrm{CM}$ characteristics by a series of research projects on $\mathrm{CM}$ diagnostics.

The HKAIM expanded to over 700 members inclusive of doctors, nurses, therapists, pharmacists, scientists and students. A good fraction of the members on the HKSAR Chinese Medicine Development Committee formed in 2013 were members of HKAIM. The Association has worked very hard to promote a dialogue between the practitioners in both fields. Indirectly, through its key members, the HKAIM has also impacted policies at the universities, the Department of Health (DH), the HA, and the FHB.

\section{Development of IM in Hong Kong}

With the HKAIM as the formal common platform for WM and CM, there came increasing cross-disciplinary interaction and development. The SARS epidemic in 2003 made an important milestone for such crossdisciplinary fertilization. While Hong Kong was baffled by a life-threatening infection for which WM offered no definitive medical solution, news of good recovery from SARS traveled in from Mainland China. The HKAIM invited while the HA engaged the CM professors with SARS experience in Guangzhou to travel to Hong Kong to share their expertise. Their presence in the local scene ${ }^{(4)}$ enabled the setup of protocols incorporating $\mathrm{CM}$ as a research arm in the HA service, leading to the establishment of the postSARS tripartite $\mathrm{CM}$ clinics run by the $\mathrm{HA}$, universities and non-governmental organizations (NGO) as well as the establishment of CMCTR working to provide evidencebased $\mathrm{CM}$ service and training for $\mathrm{CM}$ graduates, targeting to enhance healthcare delivery in the public sector.

The vision for the development of IM has evolved. To start with, the mainland developed a good environment for IM through support in clinical practice, training, scientific research and policy making. Awareness of different local and global policies and systems started our Hong Kong IM approach. It involves quality practice, mutual development and research projects from different disciplines. The system, recognized by mainland officials, is to bring the two streams of CM and WM together. The following describes some major IM activities.

\section{Education: Magnifying CM Expertise and WM Understanding \\ From CM to WM}

Degree programmes were set up at the HKBU in 1998, and subsequently at the HKU and the CUHK. Master degree courses were offered. Doctor of Medicine, Doctor of Phylosophy and post Doctorate positions are mostly for CM research.

The Chinese Medicine Council of Hong Kong has run a continuing medical education programme since 2004 . The HKAIM, often in collaboration with the HA, has been active in providing educational seminars in which WM specialists and $\mathrm{CM}$ experts together expound on how diseases can be best managed. CM associations and UGC-funded programmes delivering CM lectures at different universities, as well as various conferences and seminars, would nowadays also invite WM specialists as speakers. Since 2009, CM graduates are offered 3-year in-service training programme at the tripartite clinics. ${ }^{(5)}$ Scholarships have also been set up to train potential leaders in CM specialties in renowned centres in Mainland China. A broader audience of $\mathrm{CM}$ and WM professionals, academics and managers have joined annual conferences of the International Conference on Modernized Chinese Medicine since 2002, those of CGCM since 2003, and seminars on CM developments held by the ITC since 2013 .

\section{From WM to CM}

To increase mutual understanding, it is imperative that $\mathrm{CM}$ practitioners acquire more knowledge about WM practice. In Mainland China, IM started with WM doctors learning CM in the 1950s. In Hong Kong, the part-time $\mathrm{CM}$ degree courses organized by School of Professional and Continuing Education of HKU in the 1990s played a significant role in grooming certain WM doctors who had attended these courses as IM leaders. The HA organizes CM certificate courses for WM doctors to promote CM-WM communication. In 2017, the HKAIM made a breakthrough in promoting clinical IM via interactive workshops in which a mixed WM-CM panel offered expert-led clinical and case-illustrative discussions while one third of the audience were WM practitioners.

Since 2003, the HKAIM, in collaboration with the $\mathrm{HA}$, organize conferences involving both local and global academicians as speakers, covering a broad range of topics 
including cancer, infectious diseases, cerebrovascular and cardiovascular diseases, skin diseases, pain acumoxa, acupuncture, geriatrics, chronic diseases, CM hospital operation, and IM. Since 2013, the HKAIM has run courses, with good response, as these courses enable the $\mathrm{CM}$ practitioners to learn directly from WM experts so that the CM practitioners could understand the whole spectrum of WM.

\section{Services}

\section{Service Provision}

The HA, tasked with developing one $\mathrm{CM}$ clinic in each district since 2000, built up tripartite collaboration involving the $\mathrm{HA}$, the universities, and NGOs and formed the 18 CMCTRs. Clinical IM protocols were developed in Kwong Wah Hospital (KWH). CM services were all along provided only in clinics. The first primary care clinic with CM-WM joint consultation was started by HKBU in Queen Elizabeth Hospital in 2006.

Since 2007, HKBU has collaborated with the Hong Kong Anti-Cancer Society to provide integrative CM and WM service, including but not limited to stroke rehabilitation, pain syndrome, and late-stage cancer with 6 inpatient beds in the Nam Long Hospital, which was subsequently transformed as the Hong Kong Anti-Cancer Society Jockey Club Cancer Rehabilitation Centre. These two institutions started the Dr \& Mrs Michael SK Mak Integrated Chemotherapy Centre for cancer patients in 2012.

Within a decade, there has been much IM development: geriatric services in Princess Margaret Hospital, Fung Yiu King Hospital, Shatin Hospital, and Haven of Hope Hospital; the Duchess of Kent Children's Hospital introduced neurological rehabilitation. ${ }^{(5)}$ This movement later expanded to allow WM inpatients to request for referral for CM services with parallel or sequential CM/WM consultation and intervention. More than 20 hospitals are now providing some degree of such service. Evidence-based medicine (EBM) is being held as the key principle that the Government steers the development of services integrating CM and WM.

IM for specific diseases include early treatment for mental health at the Tang Shiu Kin CMCTR collaborating with clinical psychologists from Queen Mary Hospital and CM practitioners trained at Kowloon Hospital. Acute low back pain was piloted at the Accident and Emergency Department in Pok Oi Hospital. In response to the growing demand for pain management in cancer patients, multiple initiatives have been undertaken. At the Prince of Wales Hospital Cancer Centre, acupuncture has been used for pain in advanced cancer. The Ha Kwai Chung CMCTR, along with the Cancer Centre of $\mathrm{PMH}$, explored IM services to provide comprehensive services for cancer patients. Ngau Tau Kok Chinese Medicine Centre and the United Christian Hospital collaborated in trials of $\mathrm{CM}$ and WM for treating chronic pain with acupuncture and/or massage. Finally, for low back pain, stroke and cancer, IM service protocols were upgraded via the Integrated Chinese-Western Medicine (ICWM) Pilot Programme by the HA in 2014.

The year 2013 marked the Government's initiation of the CM hospital. It will operate under the collaboration between CM and WM. In 2018, the Chinese Medicine Hospital Project Office was set up under FHB to plan and develop the CM Hospital for 400 beds.

\section{Patient-Driven IM Service and Referral Systems}

Study in Hong Kong showed that, over the years, some $30 \%-40 \%$ of chronically ill patients use both CM and WM at the same time. ${ }^{(6)}$ In this patient-driven clinical setting, $\mathrm{CM}$ practitioners not only use techniques from traditional $\mathrm{CM}$ theory and practice but also utilize modern $\mathrm{CM}$ research results. In the $\mathrm{CM}$ clinic of the $\mathrm{HKU}$, for example, CM oncologists will take into account patients' WM data and will use $\mathrm{CM}$ and coordinate herbal actions according to the tumor types, stages, and stage of WM treatment.

For the Hong Kong Government, CM has to be promoted on the basis of evidence-based CM. For frontline practitioners of both disciplines, mutual respect and acceptance arise from mutual trust built during interactions in a conducive system/environment. In 2014, the Hong Kong Institute of Integrative Medicine (HKIIM) embarked on IM clinics staffed by both CM and WM professionals; mutual referrals are facilitated by specialized nurses.

\section{Research}

Interest of WM workers in CM started early since the 1970's. Acupuncture for heroin addicts pioneered original research. That for induction of labor in post term pregnancies was studied. Development of mouse models for $\mathrm{CM}$ patterns in spinal condition and herbal medicine for prevention of amputation in diabetic gangrene were other pioneering examples.

\section{Seeking Good Evidence-Based CM}

There are some pillars to build up EBM in IM. For one, evidence of $\mathrm{CM}$ effectiveness for diseases needs to be demonstrated. For the major disease burden, the HA commissions systematic reviews of RCTs and new 
RCTs, resulting in many publications in English peerreviewed journals. Since 2009, research training courses have been offered to CMPs in CMCTRs to enhance their basic knowledge.

Besides, clinical research activities need to be promoted. HKBU started clinical research on IBS, functional constipation, rheumatoid arthritis and Parkinson's disease. CUHK conducts research on diabetes, hepatitis, lung cancer, asthma, rheumatism, carpal tunnel syndrome, gallstone, multiple sclerosis and functional gastrointestinal diseases. HKU, combining principles and methods of traditional and modern medicine, works on the prevention and treatment of cardiovascular and cerebrovascular diseases, neuropsychiatric diseases, chronic pain, and malignant tumors as well as acupuncture and its effectiveness and mechanisms. Other universities in Hong Kong also establish their CM institutes. Work is also done for modernizing CM. HKUST with years of neuroscience research worked up $>100$ herbs for neurodegenerative diseases, Parkinson's disease and depression. After 2010, the HA has established a mechanism to commission and review CM research projects. Topics include CM-WM interactions, obesity, traumatic brain injury and insomnia, dysphagia after swallowing, etc.

\section{Promoting Evidence-Based Practice of CM}

Another pillar is at the practice level. The CM Research Practical Training programme was launched to enhance CM practitioners' competency and CMCTRs' capacity for evidence-based practice. Since 2017, CUHK's Integrative Medicine Clinical Evidence Portal ${ }^{(7)}$ offers to practitioners a search engine for evidenced-based CM usage and practice, as Asia's first CM clinical evidence on-line portal, gathering studies from around the world. Placing CM with WM on the same electronic platform for HA data coding in the Clinical Management Information System using the system from the mainland, it has impacts by contributing significantly in the development of the new electronic version of the 2019 International Classification of Diseases (ICD 11) after being put forward to WHO in 2011.

Details are necessary for safer CM-WM collaboration. A CM Toxicology Laboratory was built in $\mathrm{PMH}$ to support or refute diagnosis of herbal toxicity. The HA e-Knowledge Gateway Intranet was enriched with a 'herb-drug interaction database' arising from commissioned scientific reviews. Hong Kong Government in 2011 formed the Committee on Research and Development of Chinese Medicine. ITC seminars in conjunction with $\mathrm{HA}$ and $\mathrm{DH}$ discussed $\mathrm{CM}$ -
WM collaboration, exchanging EBM experiences and researches on $\mathrm{CM}$ and WM complementarity.

\section{Opening Wider Perspectives for CM Research}

The year 2014 was a special year for IM advancement. HKIIM of CUHK, the ICWM Pilot Programme for HA in-patients and the CM hospital were started. The Endowed Professor in IM in HKU was established earlier.

Hong Kong Government has reserved a piece of land at Tseung Kwan $\mathrm{O}$ for the CM hospital with facilities to support teaching, clinical practice and scientific research of the SCMs under the three Universities. The Chinese Medicine Hospital Project Office set up in 2018 is dedicated to its development. The CM hospital was initially proposed by an NGO as its building is in the town centre in Tsimshatsui in Kowloon, Hong Kong. Dr. Yu ECL worked once in this place and previously initiated the elders of the NGO through a university for a CM hospital setup. Following SCM of HKBU establishing a centre in that building, through Prof. Bian ZX then, it actively worked for housing the CM hospital there. Land grants however were a problem. Finally the Hong Kong Government responded and finalized it in the Tseung Kwan O site. For this, Dr. Ko WM was taking lead during his time as the Secretary of FHB.

HKIIM developed a comprehensive platform with research and development, clinical service and teaching. Collaboration between different disciplines and scholars from different areas under models of safe and effective IM treatment is expected to achieve breakthrough results. On common chronic conditions with unmet needs in treatment, including functional gastrointestinal disorders, neurodegenerative diseases and palliative medicine, IM clinical trials were initiated. The Analytic and Clinical Cooperative Laboratory for Integrative Medicine (ACCLAIM) is a joint platform for scientists and clinicians in sharing information technology, data analysis, and clinical research for the advancement of evidence-based IM. It has a focus on downstream applied clinical research, with translational deliverables and big-data applications in clinical trials, building on an established international collaboration network.

The ICWM Pilot Programme cumulates experience on ICWM in-patient care for realizing a model/ framework at the system level. With protocols for treating defined target patient groups basing on the best available evidence, and with treatment objectives of CM and WM streams defined and complementing each other for patient care, treatment outcomes can be monitored for overall evaluation. A clinical 
framework was developed to guide disease selection, service scope, clinical management and managing clinical risks. An operation framework functions to guide patient flow, organization of care processes and development of infrastructure systems. Three disease areas including stroke care, low back pain care and cancer palliative care were chosen, being joined by 3 and later 7 public hospitals. The phase III programme launched in 2018 additionally includes shoulder and neck pain care. The experiences would be of value for the further development of ICWM in Hong Kong.

\section{Supported with Funders}

The Innovational and Technology Fund of ITC, the Health and Medical Research Fund (HMRF), and the Research Grants Council (RGC) provide good funding sources. Researches range from studies on attitudes and behaviors towards the use of $\mathrm{CM}$, integrated approach to treatment using CM and WM for diseases, understanding the mechanism and application of herbs in diseases, and better designs to study the efficacy of CM and IM, and better rehabilitative and palliative management. Another direction is to develop new useful CM products, and finding active ingredients from herbs. Funders would emphasize the purpose of such researches, the basic and clinical research contributions, and the impact to combine the research outputs into practice. The better defined the local needs together with success to advance into international domains will draw better support.

\section{Future: Applying IM For Community Use}

The year 2018 marked another important milestone for the Hong Kong Government in setting up a dedicated $\mathrm{CM}$ unit to oversee the development of $\mathrm{CM}$ in Hong Kong.

While much has been done in public hospitals, it is in the community, where $\mathrm{CM}$ can play an advantageous role for chronic diseases, and side by side CM-WM practice can bring people better health service. Facilitative details are needed for WM-CM coordination and communication. Since 2015, HKAIM started an Integrative Joint Organizational Platform, with the objective to build a conducive environment for CM-WM collaborative practice. ${ }^{(8)}$ It focuses on using quantitative and qualitative inter-professional approaches as the basis for exchanging experience and ideas to compose good details for collaboration while creating opportunities and avenues of CM-WM collaboration in the community.

HKAIM public education went into the media with Radio Television Hong Kong in 2004, with alternating WM and $\mathrm{CM}$ experts in programmes on clinical problems. It was restarted as a long series of weekly programmes after 2013, presenting evidenced knowledge of herbal formulae and $\mathrm{CM} / \mathrm{IM}$ practices to the public, as these capture the interests of WM doctors. An internet platform e-Knowledge Gateway for public information, education and research was built by HA in 2011 and revised in 2015.

In Hong Kong, integrating $\mathrm{CM}$ and WM is to have the two streams collaborating, by jointly determining the diagnosis and observing clinical management of patients. The purpose is to combine the advantages of the two medical disciplines, strengthen the results of clinical curative effects and minimize the side effects in the course of medical treatment. Then effective applications may make possible the formulation of a plan that is most suitable for the patient to accelerate recovery.

\section{Acknowledgement}

This article was based on a Dr. Yu ECL's paper written as HKAIM published an October issue on IM in Hong Kong. ${ }^{(9)}$ We thank with much appreciation for the help and contributions in writings from WONG TAAM Vivian, CHEUNG Wai-lun, WU Justin, FENG Yi-bin, and CHOW Shew-ping who provided stimulating informative guides on developments in $\mathrm{HA}$ and the Universities.

The author apologies for limitations in embrasure of modernization of CM and herb-pharmacy, of nursing and paramedical developments, the herbal industry, and other research and funding institutions.

\section{REFERENCES}

1. Starling A, Ho FCS, Luke LL, Tso SC, Yu ECL. Plague, SARS and the story of medicine in Hong Kong, Hong Kong Museum of Medical Sciences Society, Chapter 5. Health-Care Issues in, A Changing Society; 2006:245-280.

2. Chan CS, Chow SP. Electroacupuncture in the treatment of posttraumatic sympathetic dystrophy(Sudeck's atrophy). Br J Anaesth 1981;53:899-902.

3. Chow SP, Yeung HW, Law LK, Chan TM, Lau C. The effect of Davalina Orientalis on bone healing - a preliminary report. Am J Chin Med 1982;10:101-106.

4. Chan JCK, Wong VCW, eds. Challenges of SARS. Singapore: Saunders/Elsevier; 2006.

5. http://www.info.gov.hk/gia/general/200904/22/P200904220180.htm

6. Leong $\mathrm{CH}$, Ko WM, Liu L, Mak SC, Fung L, et al. Development of Chinese medicine in Hong Kong. A background report. 2010:1-46.

7. Integrative Medicine Clinical Evidence Portal, The Chinese University of Hong Kong. Available at: http://www.hkiim.cuhk.edu.hk/ceim/tc

8. Integrative Joint Organizational Platform IJOP 2017 Report. Hong Kong: Hong Kong Association for Integration of Chinese-Western Medicine.

9. Yu ECL. History of integrative medicine in Hong Kong. Hong Kong Med Diary 2018;24(10):4-12.

(Accepted January 5, 2019) Edited by YUAN Lin 